\title{
Induction in a Small Loop Moving With a Magnetostatic Dipole Toward a Conducting Half Space
}

\author{
Martin B. Kraichman \\ Contribution From U.S. Naval Ordnance Laboratory, White Oak, Silver Spring, Maryland
}

(Received March 16, 1962; revised May 23, 1962)

\begin{abstract}
A formal solution is obtained for the magnetic field produced by a vertical magnetostatic dipole which moves in free space at a constant velocity along the normal to a conducting half space. When the velocity of the dipole is much less than that of light, the field in free space is governed by Laplace's equation, and that in the conducting half space by the diffusion equation. An expression in closed form is derived for the voltage induced in a small loop moving with the dipole. For certain values of the parameters, simplified expressions are obtained for the induction. Numerical results are presented for certain cases.
\end{abstract}

\section{Introduction}

The problem of a stationary vertical oscillating magnetic dipole above a conducting ground has been discussed by Wait $[1951,1953]$. The analagous problem of a vertical magnetostatic dipole approaching a conducting half space is considered in the present paper. If the dipole moves with a constant velocity which is much smaller than that of light, then the magnetic field in free space will satisfy Laplace's equation while the field in the conducting region will satisfy the diffusion equation. Formal solutions are obtained for the magnetic field in the two regions.

It is of interest to find an expression for the voltage induced in a small loop moving with the dipole. Such a result might have application in the design of a device for measuring the close proximity of a vehicle to a conducting surface or for measuring the velocity of a vehicle just before impact with such a surface. The solution for the reflected vertical component of the magnetic flux density in free space is used to find an expression in closed form for the induction. This result simplifies considerably for certain values of the parameters. Numerical results are presented for certain cases.

\section{Formal Solution}

With reference to the coordinate system shown in figure 1, the conducting medium of conductivity $\sigma$, permeability $\mu$, and permittivity $\epsilon$ occupies the half space defined by all negative values of $z$. The upper half space has the characteristics of free space, namely, zero conductivity, permeability $\mu_{0}$, and permittivity $\epsilon_{0}$. A magnetostatic dipole of moment $m$ moves toward the conducting half space with a constant velocity $v$ along the $z$ axis. The dipole is situated instantaneously on the $z$ axis at $z=d$ and is oriented in the positive $z$ direction. The field point $P$, located at $\rho, \phi, z$, in cylindrical coordinates, is a distance $r$ from the dipole. The vector magnetic flux density $\bar{B}$ can be represented everywhere in terms of a magnetic vector potential $\bar{A}$.

Thus,

$$
\bar{B}=\nabla \times \bar{A}
$$

where $\bar{A}$, if $\imath \ll c(c=$ free space velocity of light), satisfies

$$
\nabla^{2} \bar{A}=0
$$

for $z>0$, and

$$
\nabla^{2} \bar{A}=\mu \sigma \frac{\partial \bar{A}}{\partial t}
$$

for $z<0$.

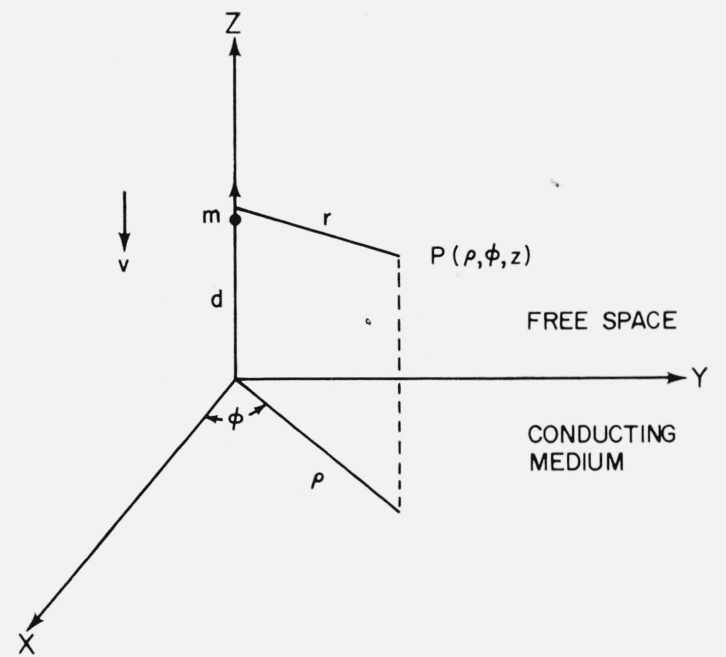

Figure 1. Magnetostatic dipole moving toward a conducting half space. 
In the region $z>0$, the total vector potential is assumed to consist of a primary potential $\bar{A}_{0}$ plus a reflected potential $\bar{A}_{1}$. The primary vector potential of the magnetostatic dipole, shown in figure 1, has only a $\phi$ component which is given by

$$
A_{\phi_{0}}=-m \frac{\mu_{0}}{4 \pi} \frac{\partial}{\partial \rho}\left(\frac{1}{r}\right)
$$

The quantity $\frac{1}{r}$ may be expressed [Smythe, 1950] as

$$
\frac{1}{r}=\int_{0}^{\infty} e^{-k|d-z|} \boldsymbol{J}_{0}(k \rho) d k,
$$

where $r=\left[\rho^{2}+(d-z)^{2}\right]^{1 / 2}$. The primary potential then reads

$$
A_{\phi_{0}}=m \frac{\mu_{0}}{4 \pi} \int_{0}^{\infty} e^{-k|d-k|} J_{1}(k \rho) k d k, \quad(z>0) .
$$

Similarly, the reflected potential in the upper half space, $A_{\phi_{1}}$, and the potential in the conducting half space, $A_{\phi_{2}}$, may be written as

$$
A_{\phi_{1}}=m \frac{\mu_{0}}{4 \pi} \int_{0}^{\infty} f_{1}(k) e^{-k(d+z)} J_{1}(k \rho) k d k, \quad(z>0)
$$

and

$$
A_{\phi_{2}}=m \frac{\mu_{0}}{4 \pi} \int_{0}^{\infty} f_{2}(k) e^{\alpha(k) z} e^{-k d} J_{1}(k \rho) k d k, \quad(z<0),
$$

respectively.

The functions $\alpha(k), f_{1}(k)$, and $f_{2}(k)$ are determined from (2) and the boundary conditions at $z=0$ for the normal magnetic flux density and the tangential magnetic intensity.

Upon substituting the expression for $A_{\phi_{2}}$ into (3), the relationship

$$
\alpha^{2}=k^{2}+k \mu \sigma v
$$

is obtained where $v$, the velocity of the dipole, equals $-\frac{\partial d}{\partial t}$. Application of the continuity conditions for the magnetic field at the boundary $z=0$ yields

$$
A_{\phi_{0}}+A_{\phi_{1}}=A_{\phi_{2}}, \quad \text { (normal } \bar{B} \text { continuous) }
$$

and

$$
\frac{A_{\phi_{0}}}{\mu_{0}}+\frac{A_{\phi_{1}}}{\mu_{0}}=\frac{A_{\phi_{2}}}{\mu}, \quad \text { (tangential } \bar{H} \text { continuous). }
$$

The functions $f_{1}(k)$ and $f_{2}(k)$ are evaluated by substituting the potentials given by (6), (7), and (8) into (10) and (11). This results in

$$
f_{1}(k)=\frac{\mu k-\mu_{0} \alpha}{\mu k+\mu_{0} \alpha}
$$

and

$$
f_{2}(k)=\frac{2 \mu k}{\mu k+\mu_{0} \alpha} .
$$

The components of the magnetic flux density in each region are then found from

and

$$
B_{z}=\frac{1}{\rho} \frac{\partial}{\partial \rho}\left(\rho A_{\phi}\right)
$$

$$
B_{\rho}=-\frac{\partial A_{\phi}}{\partial z}
$$

\section{Voltage Induced in Loop Moving With Dipole}

\subsection{Solution in Closed Form}

An interesting application of the solution for the reflected magnetic flux density in free space is the determination of the voltage induced in a small, single-turn loop moving with the dipole along the $z$ axis. If the axis of the loop is vertical and the permeability of both regions is taken as $\mu_{0}$, the voltage per unit loop area, $V$, is given by

$$
V=-\frac{d B_{z_{1}}}{d t}=-\frac{m v \mu_{0}}{2 \pi} \int_{0}^{\infty}\left[\frac{1-\left(\frac{p^{2}}{k}+1\right)^{1 / 2}}{1+\left(\frac{p^{2}}{k}+1\right)^{1 / 2}}\right] e^{-2 k d} k^{3} d k,
$$

where $p^{2}=\mu_{0} \sigma v$. The integral in (16) is evaluated in appendix 1 and can be written as

$$
\begin{gathered}
V=\frac{m v \mu_{0}}{2 \pi}\left[\frac{3}{2 p^{2} d^{5}}+\frac{3}{8 d^{4}}+2 p^{8} e^{\frac{h}{2}}\left(-\frac{1}{4 h}+\frac{9}{8 h^{2}}-\frac{3}{h^{3}}\right) K_{0}\left(\frac{h}{2}\right)\right. \\
\left.+2 p^{8} e^{\frac{h}{2}}\left(\frac{1}{4 h}-\frac{11}{8 h^{2}}+\frac{9}{2 h^{3}}-\frac{12}{h^{4}}\right) K_{1}\left(\frac{h}{2}\right)\right],
\end{gathered}
$$

where $h=2 p^{2} d$.

\subsection{Solution for $\mathrm{h} \ll 1$}

For $h \ll 1$, the expression for the voltage given by (17) is not useful for numerical computation due to the fact that the sum of the first two terms is opposite in sign and very nearly equal to the sum of the third and fourth terms. A more convenient expression may be derived by substituting $h=2 p^{2} d$ and $x=\frac{k}{p^{2}}$ in the integral in (16). The equation then reads

$$
\begin{aligned}
V=m v \frac{\mu_{0}}{2 \pi}\left[\int_{0}^{\infty}\right. & p^{8} e^{-h x} x^{3} d x \\
& \left.+\int_{0}^{\infty} 2 p^{8} x^{3}\left(x-\left[x^{2}+x\right]^{1 / 2}\right) e^{-h x} d x\right] .
\end{aligned}
$$


Evaluation of the first integral in (18) is simple and yields $\frac{3}{16 \pi} \frac{m v \mu_{0}}{d^{4}}$. The second integral is evaluated in appendix 2 by neglecting terms of the order of $h^{3}$ and higher. The expression for the voltage then simplifies to

$$
V=\frac{m v \mu_{0} p^{2}}{32 \pi d^{3}}=\frac{m \sigma \mu_{0}^{2} v^{2}}{32 \pi d^{3}} .
$$

\subsection{Special Case for $\sigma=\infty$}

For the case where the conductivity of the lower half space becomes infinite, the solution for the voltage may be obtained by either the method of images or by using (16). The resulting expression is

$$
V=\frac{3}{16 \pi} m \mu_{0} \frac{v}{d^{4}},(\sigma=\infty) .
$$

\section{Discussion of Numerical Results}

When $h \ll 1$, or equivalently when $\sigma v d \ll 1 / 8 \times 10^{7}$, the expression for the voltage given by (19) may be used. Numerical results using this expression are presented in figures 2 and 3 for the case where $m=1$ $\mathrm{amp} / \mathrm{m}^{2}$. This value of the moment corresponds to that of $1 \mathrm{~cm}^{3}$ of Alinco 5 in which there is an

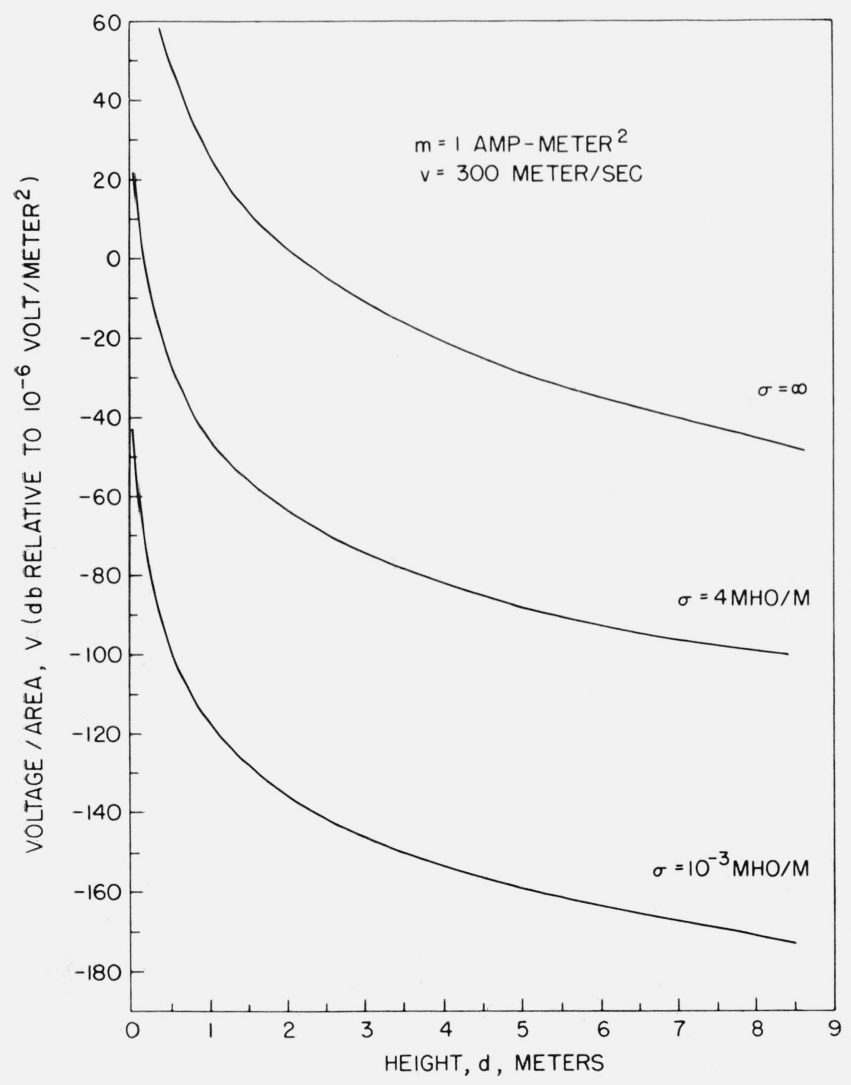

Figure 2. Induced voltage as a function of height above a conducting half space. average flux density of 1.2 webers $/ \mathrm{m}^{2}$. In figure 2 , the voltage induced in a loop of unit area is plotted as a function of the height of the magnet above surfaces of different conductivity. The velocity of the loop and magnet is taken as $300 \mathrm{~m} / \mathrm{sec}$. Figure 3 shows the voltage per unit area as a function of velocity for various half space conductivities. The values of induction in this figure correspond to a one meter height of the loop and magnet above the conducting surface.

The numerical results plotted in fioures 2 and 3 show the induction to be quite small. Usable voltages could be obtained just above earth or sea water by employing large magnets and small multiturn loops moving with velocities near Mach 1 .

\section{Appendix 1}

Using the substitution $x=\frac{k}{p^{2}}$, the integral in (16) is written as

$$
\begin{array}{r}
V=m v \frac{\mu_{0}}{2 \pi} \int_{0}^{\infty}\left[2 p^{8} e^{-2 x p^{2} d} x^{4}-2 p^{8}\left(x^{2}+x\right)^{1 / 2} e^{-2 x p^{2} d} x^{3}\right. \\
\left.+p^{8} e^{-2 x p^{2} t} x^{3}\right] d x .
\end{array}
$$

The first and third terms are readily integrated.

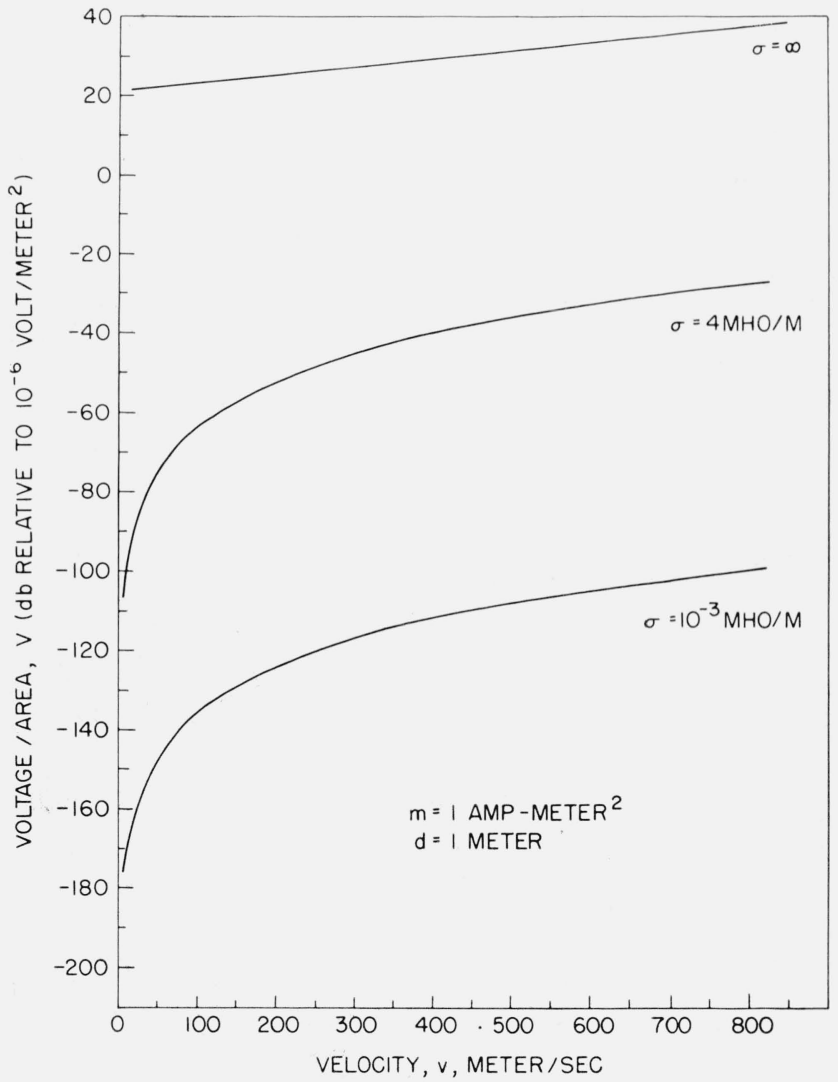

FIGURE 3. Induced voltage as a function of velocity towards a conducting half space. 
Thus,

$$
\begin{aligned}
\frac{m v \mu_{0}}{2 \pi} \int_{0}^{\infty}\left[2 p^{8} e^{-2 x p^{2} d} x^{4}+p^{8} e^{-2 x p^{2} d} x^{3}\right] d x & \\
& =\frac{m v \mu_{0}}{2 \pi}\left[\frac{3}{2 p^{2} d^{5}}+\frac{3}{8 d^{4}}\right] .
\end{aligned}
$$

The second integral can be written as

$$
\begin{aligned}
\frac{-m v \mu_{0} p^{8}}{\pi} \int_{0}^{\infty} x^{3}\left(x^{2}+x\right)^{1 / 2} e^{-h x} d x & \\
= & \frac{m v \mu_{0} p^{8}}{\pi} \frac{d^{3}}{d h^{3}} \int_{0}^{\infty}\left(x^{2}+x\right)^{1 / 2} e^{-h x} d x,
\end{aligned}
$$

where $h=2 p^{2} d$. If the substitution $x=\frac{1}{2} \cosh \beta-\frac{1}{2}$ is made in the integral on the right side of (A3), the integral reads

$$
\begin{aligned}
\int_{0}^{\infty}\left(x^{2}+x\right)^{1 / 2} e^{-h x} d x & =\frac{1}{4} \int_{0}^{\infty} \sinh ^{2} \beta e^{-\frac{h}{2}(\cosh \beta-1)} d \beta \\
& =\frac{1}{8} e^{\frac{h}{2}} \int_{0}^{\infty}(\cosh 2 \beta-1) e^{-\frac{h}{2} \cosh \beta} d \beta .
\end{aligned}
$$

Since $K_{n}(u)$, the modified Bessel function of the second kind, is defined [Erdélyi, ed., 1953] by

$$
K_{n}(u)=\int_{0}^{\infty} e^{-u \cosh \beta} \cosh n \beta d \beta,(u>0),
$$

(A4) can be written as

$$
\begin{aligned}
\int_{0}^{\infty}\left(x^{2}+x\right)^{1 / 2} e^{-h x} d x & =\frac{1}{8} e^{\frac{h}{2}}\left[K_{2}\left(\frac{h}{2}\right)-K_{0}\left(\frac{h}{2}\right)\right] \\
& =\frac{1}{2 h} e^{\frac{h}{2}} K_{1}\left(\frac{h}{2}\right) .
\end{aligned}
$$

The third derivative with respect to $h$ of the integral in (A5) is obtained by using the following recurrence relations:

$$
\begin{aligned}
\frac{2}{u} K_{1}(u) & =K_{2}(u)-K_{0}(u) \\
K_{0}^{\prime}(u) & =-K_{1}(u) \\
K_{1}^{\prime}(u) & =-\left[K_{0}(u)+\frac{1}{u} K_{1}(u)\right],
\end{aligned}
$$

where the primed quantities represent derivatives with respect to $u$. The second integral of (A1) is finally expressed as

$$
\begin{aligned}
& \frac{-m v \mu_{0} p^{8}}{\pi} \int_{0}^{\infty} x^{3}\left(x^{2}+x\right)^{1 / 2} e^{-h x} d x \\
& =\frac{m v \mu_{0} p^{8}}{\pi} e^{\frac{h}{2}}\left[\left(-\frac{1}{4 h}+\frac{9}{8 h^{2}}-\frac{3}{h^{3}}\right) K_{0}\left(\frac{h}{2}\right)\right. \\
& \left.\quad+\left(\frac{1}{4 h}-\frac{11}{8 h^{2}}+\frac{9}{2 h^{3}}-\frac{12}{h^{4}}\right) K_{1}\left(\frac{h}{2}\right)\right] .
\end{aligned}
$$

\section{Appendix 2}

By letting $t=h x$, the second integral in (18) can be written as

$$
\begin{aligned}
& \frac{m v \mu_{0} p^{8}}{\pi} \int_{0}^{\infty} x^{3}\left[x-\left(x^{2}+x\right)^{1 / 2}\right] e^{-h x} d x \\
& =\frac{m v \mu_{0} p^{8}}{\pi h^{5}} \int_{0}^{\infty} t^{3}\left[t-\left(t^{2}+t h\right)^{1 / 2} e^{-t} d t .\right.
\end{aligned}
$$

The integral on the right of $(A 7)$ is written in two parts, namely,

and

$$
I_{1}=\int_{0}^{h} t^{3}\left[t-\left(t^{2}+t h\right)^{1 / 2}\right] e^{-t} d t
$$

$$
I_{2}=\int_{h}^{\infty} t^{3}\left[t-\left(t^{2}+t h\right)^{1 / 2}\right] e^{-t} d t
$$

The $\left|I_{1}\right|$ is at most of the order $h^{5}$ since

$$
\begin{aligned}
\left|I_{1}\right|=\left|\int_{0}^{h} t^{3}\left[t-\left(t^{2}+t h\right)^{1 / 2}\right] e^{-t} d t\right| & <\int_{0}^{h} t^{3}\left(t-2^{1 / 2} h\right) d t \\
& =\frac{h^{5}}{5}-\frac{2^{1 / 2} h^{5}}{4} .
\end{aligned}
$$

For $\frac{h}{t}<1$,

$$
\begin{aligned}
\left(t^{2}+t h\right)^{1 / 2} & =t\left(1+\frac{h}{t}\right)^{1 / 2} \\
& =t\left[1+\frac{h}{2 t}-\frac{1}{8} \frac{h^{2}}{t^{2}}+\frac{1}{16} \frac{h^{3}}{t^{3}}(1+\theta(t))^{-5 / 2}\right],
\end{aligned}
$$

where $0 \leq \theta(t) \leq 1$. It follows that

$$
\begin{aligned}
I_{2} & =\int_{h}^{\infty}\left[-t^{3} \frac{h}{2}+\frac{1}{8} t^{2} h^{2}-\frac{1}{16} t h^{3}(1+\theta(t))^{-5 / 2}\right] e^{-t} d t \\
=\int_{0}^{\infty}\left[-t^{3} \frac{h}{2}+\frac{1}{8} t^{2} h^{2}\right] e^{-t} d t & \quad-\int_{0}^{h}\left[-t^{3} \frac{h}{2}+\frac{1}{8} t^{2} h^{2}\right] e^{-t} d t-R,
\end{aligned}
$$

where $R=\frac{h^{3}}{16} \int_{h}^{\infty} t[1+\theta(t)]^{-5 / 2} e^{-t} d t$ represents a remainder term. The maximum value of the remainder is found by letting $\theta(t)=0$. Thus,

$$
R<\frac{h^{3}}{16} \int_{h}^{\infty} t e^{-t} d t<\frac{h^{3}}{16} \int_{0}^{\infty} t e^{-t} d t=\frac{h^{3}}{16} .
$$

Therefore,

$$
I_{1}+I_{2}=-3 h+\frac{1}{4} h^{2}+0\left(h^{3}\right) .
$$


The second integral in (18) is then written as

$\frac{m v \mu_{0} p^{8}}{\pi} \int_{0}^{\infty} x^{3}\left[x-\left(x^{2}+x\right)^{1 / 2}\right] e^{-h x} d x=\frac{m v \mu_{0} p^{8}}{\pi}\left[-\frac{3}{h^{4}}+\frac{1}{4 h^{3}}\right]$

$$
=\frac{m v \mu_{0}}{2 \pi}\left[-\frac{3}{8 d^{4}}+\frac{p^{2}}{16 d^{3}}\right] \text {. }
$$

The author thanks Dr. A. H. Van Tuyl of the U.S. Naval Ordnance Laboratory, White Oak, Maryland for his help in evaluating the integrals considered in the appendices.

\section{References}

Erdélyi, A., ed., Higher transcendental functions, vol. II, ch. 7, p. 82, McGraw-Hill Book Co. (1953).

Smythe, W. R., Static and dynamic electricity, second ed., ch. 5, p. 179, McGraw-Hill Book Co. (1950).

Wait, J.R., The magnetic dipole over the horizontally stratified earth, Can. J. Phys. 29, 577-592 (Nov. 1951).

Wait, J.R., Radiation resistance of a small circular loop in the presence of a conducting ground, J. Appl. Phys. 24, No. 5, 646-649 (1953).

(Paper 66D6-233) 